8-2002

\title{
A Fundamental Model of Cyclic Instabilities in Thermal Barrier Systems
}

Anette M. Karlsson

Cleveland State University, a.karlsson@csuohio.edu

J. W. Hutchinson

Harvard University

A. G. Evans

University of California - Santa Barbara

Follow this and additional works at: https://engagedscholarship.csuohio.edu/enme_facpub

Part of the Mechanical Engineering Commons

How does access to this work benefit you? Let us know!

Publisher's Statement

NOTICE: this is the author's version of a work that was accepted for publication in Journal of the Mechanics and Physics of Solids. Changes resulting from the publishing process, such as peer review, editing, corrections, structural formatting, and other quality control mechanisms may not be reflected in this document. Changes may have been made to this work since it was submitted for publication. A definitive version was subsequently published in Journal of the Mechanics and Physics of Solids, 50, 8, August 2002; 10.1016/S0022-5096(02)00003-0

\section{Original Citation}

Karlsson, A. M., Hutchinson, J. W., and Evans, A. G., 2002, "A Fundamental Model of Cyclic Instabilities in Thermal Barrier Systems," Journal of the Mechanics and Physics of Solids, 50(8) pp. 1565-1589.

This Article is brought to you for free and open access by the Mechanical Engineering Department at EngagedScholarship@CSU. It has been accepted for inclusion in Mechanical Engineering Faculty Publications by an authorized administrator of EngagedScholarship@CSU. For more information, please contact library.es@csuohio.edu. 


\title{
A fundamental model of cyclic instabilities in thermal barrier systems
}

\author{
A.M. Karlsson ${ }^{\mathrm{a}, *}$, J.W. Hutchinson ${ }^{\mathrm{b}}$, A.G. Evans ${ }^{\mathrm{c}}$ \\ a Princeton Materials Institute, Princeton University, Browen Hall, 70 Prospect Avenue, Princeton, \\ NJ 08540-5211, USA \\ ${ }^{\mathrm{b}}$ Division of Engineering and Applied Science, Harvard University, Cambridge, MA 02138, USA \\ ${ }^{c}$ Department of Mechanical and Environmental Engineering, University of California, Santa Barbara, \\ CA 93106, USA
}

\section{Introduction}

Modern gas turbines used for aero-propulsion and power generation all have hot section components that consist of a single crystal $\mathrm{Ni}$ alloy with a superposed bilayer

* Corresponding author. Fax: +1-609-258-6878.

E-mail address: karlsson@princeton.edu (A.M. Karlsson). 


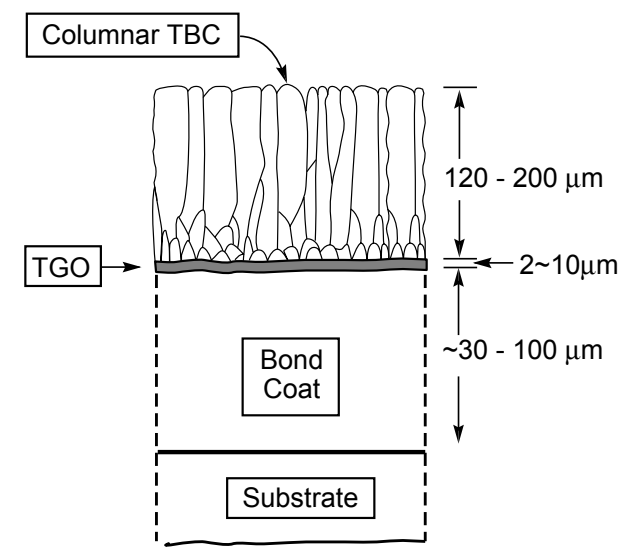

Fig. 1. A schematic of a thermal barrier system, consisting of the superalloy substrate, the bond coat, the thermally grown oxide (TGO), and the thermal barrier coating (TBC).

that provides both thermal and oxidation protection (DeMasi-Marcin and Gupta, 1994; Miller, 1984; Stiger et al., 1999; Strangman, 1985). Moreover, during operation, a third layer forms due to oxidation. The general consequence is a multilayer (thermal barrier) system that consists of four constituents (Fig. 1): (i) a thick Ni-super-alloy substrate, (ii) a relatively thin $(30-100 \mu \mathrm{m}$ thick) bond coat layer next to the substrate that imparts the oxidation protection, (iii) a very thin $(2-10 \mu \mathrm{m}$ thick) thermally grown oxide (TGO), preferably $\alpha-\mathrm{Al}_{2} \mathrm{O}_{3}$, because of its low permeability to oxygen, and (iv) a thermal barrier coating (TBC), typically comprising a 120-200 $\mu \mathrm{m}$ thick layer of yttria stabilized zirconia. The system evolves during its lifetime due to TGO-formation and the consequent influence on the other layers.

The bond coat function can be achieved using several alternative Ni-based alloys and associated microstructures. One widely used system contains $\mathrm{Ni}, \mathrm{Al}, \mathrm{Cr}$ and $\mathrm{Pt}$, chosen to exist as a single phase, based on the $\beta$ (NiAl) structure: it is referred to as Pt-aluminide. The merits of this system include its ability to form a relatively pure $\alpha-\mathrm{Al}_{2} \mathrm{O}_{3}$, which grows slowly and benefits durability. A large body of recent information has identified the predominant failure mode in such a system. It involves a cyclic displacement instability occurring in the TGO (Fig. 2), (Evans et al., 2001; Gell et al., 1999; Johnson et al., 1998; Mumm and Evans, 2000; Mumm et al., 2001; Ruud et al., 2001; Spitsberg et al., 2002; Tolpygo and Clarke, 2000; Wright and Evans, 1999). A similar instability has been observed in other systems: most notably alloys with constituents, FeCrAlY (Karlsson et al., 2002a; Rebello and Levi, 2002), used as heating elements for furnaces. The instability happens because the TGO is a thin layer subject to large levels of residual compression. The compression is caused by a combination of growth strain (converting the $\mathrm{Al}$ in the alloy to $\alpha-\mathrm{Al}_{2} \mathrm{O}_{3}$ ) and low thermal expansion coefficient that results in misfit upon cooling. The compressions reach several GPa at ambient (Karlsson and Evans, 2001; Tolpygo and Clarke, 2000). The thin TGO seeks mechanisms to relieve the compression, by means of out-of-plane displacements. The sign and magnitude of the displacements are dictated by the mechanical properties of 

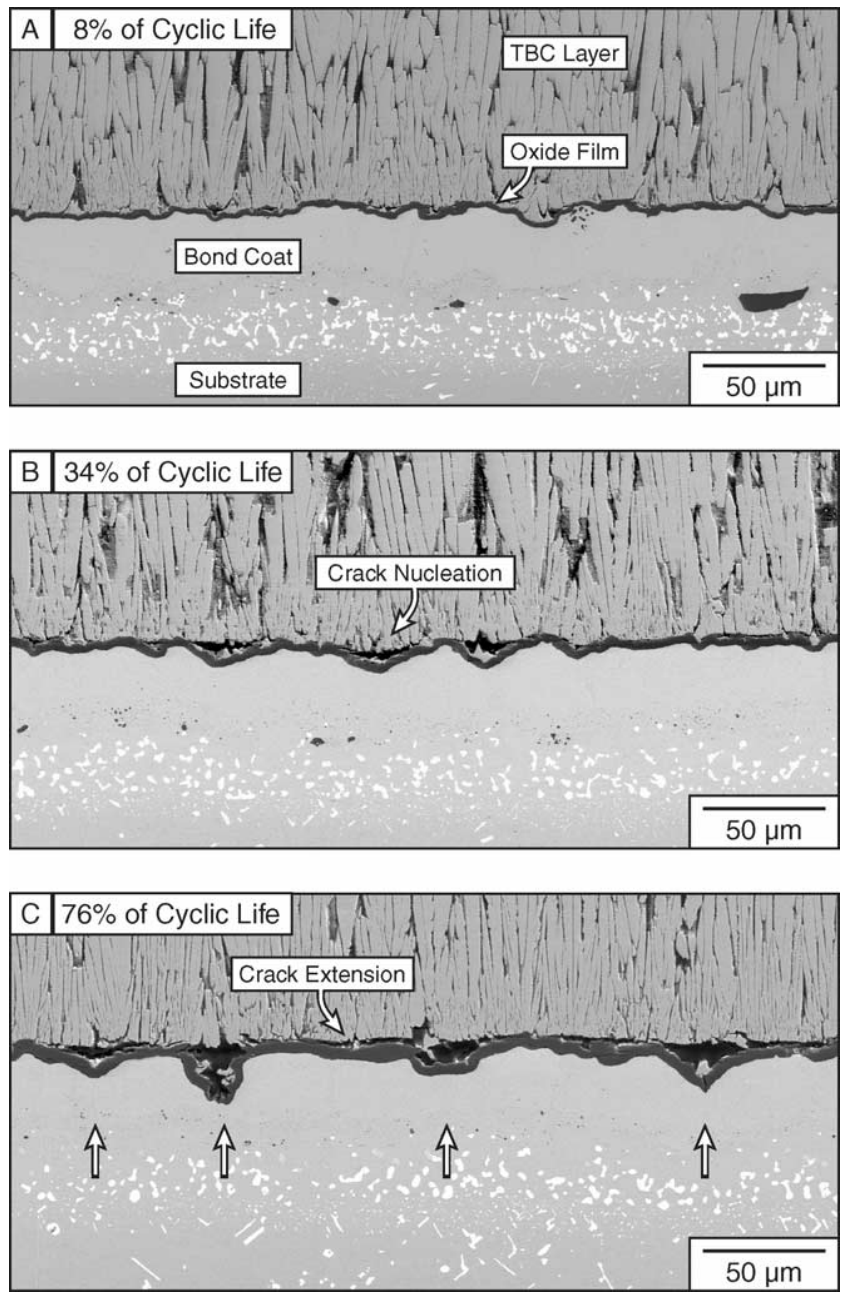

Fig. 2. An example of the displacements of the TGO that accompany thermal cycling of a thermal barrier system with a Pt-aluminide bond coat (courtesy D.R. Mumm). The arrows identify the instability sites. Note the downward displacement of the TGO layer into the bond coat as the fraction of life (indicated as percentages) increases from (a) to (c).

the bond coat and the TBC. In practice, at high temperature, the bond coat is relatively soft, while the TBC layer remains elastic. Thus, the displacements occur preferentially into the bond coat, as evident in Fig. 2. The salient experimental findings are as follows:

(i) The instability is prevalent upon thermal cycling.

(ii) The growth rate of the instability per cycle, $\mathrm{d} A / \mathrm{d} N$, decreases with the increase in the number of cycles, $N$, in a manner corresponding to the growth of the TGO, $\mathrm{d} h / \mathrm{d} N$ (Mumm et al., 2001). 


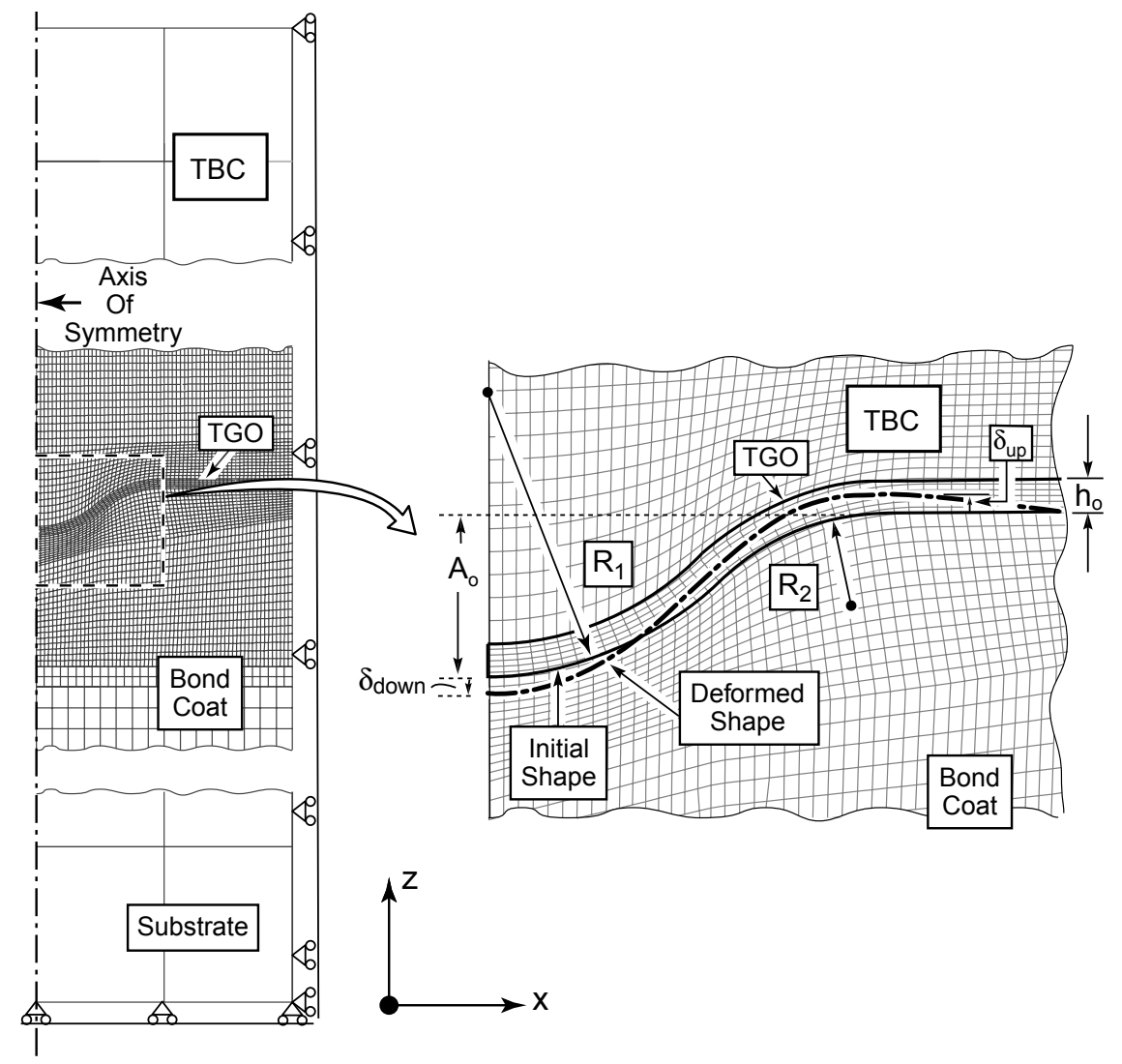

Fig. 3. Example of finite element model previously used to simulate the displacements in Fig. 2 (Karlsson et al., 2002b). The shape of the imperfection is varied by changing the ratio between the two characteristic radii $R_{2} / R_{1}$.

(iii) Removing the geometric imperfections suppresses the mechanism and enhances the durability (Spitsberg et al., 2002).

(iv) Enhancing the high temperature strength of the bond coat diminishes $\mathrm{d} h / \mathrm{d} N$ and enhances durability (Spitsberg et al., 2002).

An approach for finite element simulation that captures these primary features has been devised, using the model depicted in Fig. 3 (He et al., 2000, 2002; Karlsson and Evans, 2001; Karlsson et al., 2002a, b). The model embodies an initial geometric imperfection, inspired by the morphology found on actual turbine components (Evans et al., 2001; Gell et al., 1999; Mumm et al., 2001; Spitsberg et al., 2002; Wright and Evans, 1999). These imperfections are governed by a pre-conditioning treatment used before the TBC layer is deposited. The simulations have revealed that the propagation of the instability depends on the interaction between three different strains: cyclic plasticity 
in the bond coat, growth in the TGO, and the thermal expansion misfit between the TGO and the substrate. Moreover, the model demonstrates that the TBC layer provides constraint that inhibits the instability unless crack-like imperfections are present in the TBC (Karlsson et al., 2002; Ruud et al., 2001). While the model appears to duplicate the effects observed in the experiments, the interactions are sufficiently complex that the simulations have not been amenable to deconvolution of the influences of the properties of the individual material constituents. The intent of this study is to devise an analytic model that highlights the roles of each constituent.

Several findings from the prior simulations and experimental observations discussed above are critical. Most relevant are the plastic strains occurring in the bond coat in the vicinity of the imperfections (Ambrico et al., 2001; He et al., 2000, 2002; Karlsson and Evans, 2001; Karlsson et al., 2002a, b). These strains have a dominant radial component that facilitates the outward (downward) displacement of the instability (Fig. 4). A circumferential component also exists, but is only appreciable at the extremities, where plastic pile-up occurs (Fig. 4). Accordingly, as in analogous indentation problems (Begley et al., 1999, 2000), it is surmised that the dominant phenomena can be adequately understood by using a spherically symmetric model (Fig. 5). Indeed, there are close geometric correspondences between the present problem and the elastic/plastic indentation of a half space. It will be shown that the sphere model allows the steps in the deformation history to be solved analytically, enabling a basic understanding of some of the key findings from the simulations. Such results are regarded as an augmentation to the simulations (He et al., 2000, 2002; Karlsson and Evans, 2001; Karlsson et al., 2002a, b), which have been essential to the development of realistic models, but have not provided insight.

Prior observations (Evans et al., 2001; Mumm and Evans, 2000; Mumm et al., 2001; Ruud et al., 2001; Rebello and Levi, 2002; Spitsberg et al., 2002; Wright and Evans, 1999) and simulations (He et al., 2000, 2002; Karlsson and Evans, 2001; Karlsson et al., 2002a, b) have suggested five prerequisites for the instability. (a) The temperature must be cycled. (b) There should be a thermal expansion misfit between the TGO and substrate to generate large compressions in the TGO upon cooling. (c) The stresses induced in the bond coat must exceed its yield strength. (d) A TGO growth strain is needed that interacts with the thermal expansion misfit to vector the process. (e) An imperfection is required to initiate the instability. Beyond suggesting these basic requirements, the simulations and experiments indicate characteristic tendencies. Namely, the TGO extends primarily at curved or discontinuous segments of the interface (Karlsson et al., 2002a).

The simulations (He et al., 2000, 2002; Karlsson and Evans, 2001; Karlsson et al., $2002 \mathrm{a}, \mathrm{b}$ ) have indicated that the instability is affected primarily by lateral strains caused by new TGO forming on the internal grain boundaries, as depicted on Fig. 6. The thickening is less important. Guided by this finding, the following analyses emphasize lateral strains, designated $\Delta \varepsilon_{\mathrm{g}}$ per cycle (no thickening). This differs from a previous analysis by Ambrico et al. (2001), which examined the role of thickening.

Stress relaxation in the TGO at the highest temperature in the cycle is important and is modeled by imposing a high temperature yield strength, $\sigma_{\mathrm{Y}}^{\text {tgo }}$. At lower temperatures, the TGO is considered elastic. It will be shown that the stress in the TGO builds 

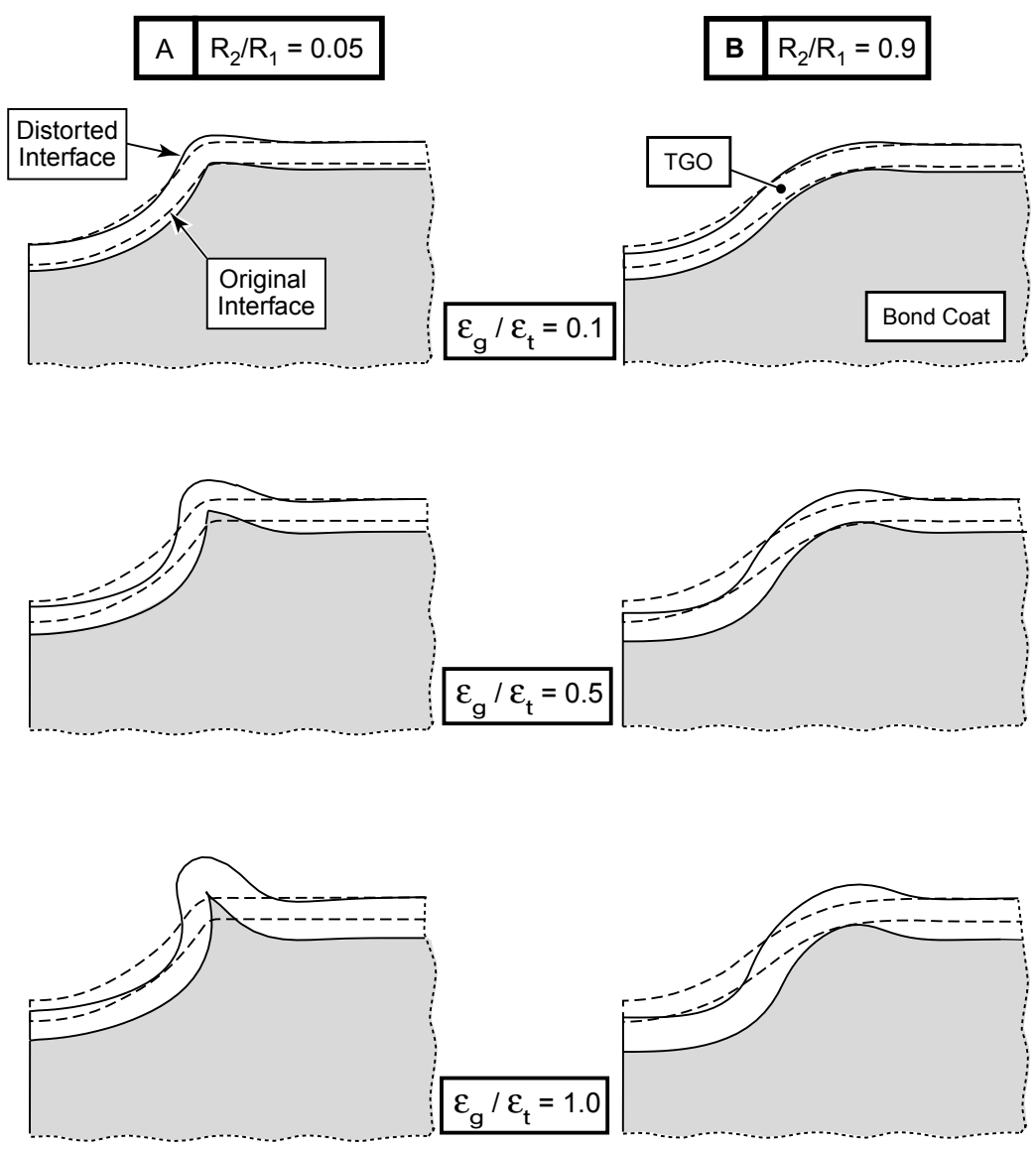

Fig. 4. Typical results from finite element simulations of the change in shape of the bond coat and TGO for two geometries (see Fig. 3) and various ratios of thickening to lengthening growth strain show that the displacement in the bond coat is down ward/radial around the base of the imperfection (Karlsson et al., 2002a). Only near the periphery are upward/circumferential displacements observed, accompanied by pile-up.

up during a "transient" stage, with the TGO remaining elastic, resulting in a stress increase per cycle. This stress elevation causes rapid displacement changes (He et al., 2000, 2002; Karlsson and Evans, 2001; Karlsson et al., 2002a, b). Thereafter, a "steadystate" develops. This happens once the stress induced in the TGO at the peak temperature attains $\sigma_{\mathrm{Y}}^{\mathrm{tgo}}$. Such yielding limits the stress in the TGO. Nevertheless, the force imposed by the TGO on the bond coat increases on a cycle-by-cycle basis. After cycling down to ambient, and upon returning to the peak temperature, the stress in the TGO is below yield and some of the lateral straining occurs while it is still elastic. Indeed, it will be shown that the essential difference between the isothermal and multicycle response found experimentally relates to the relative proportions of TGO 


\section{HEMISPHERICAL IMPERFECTION}

\section{SPHERE MODEL}

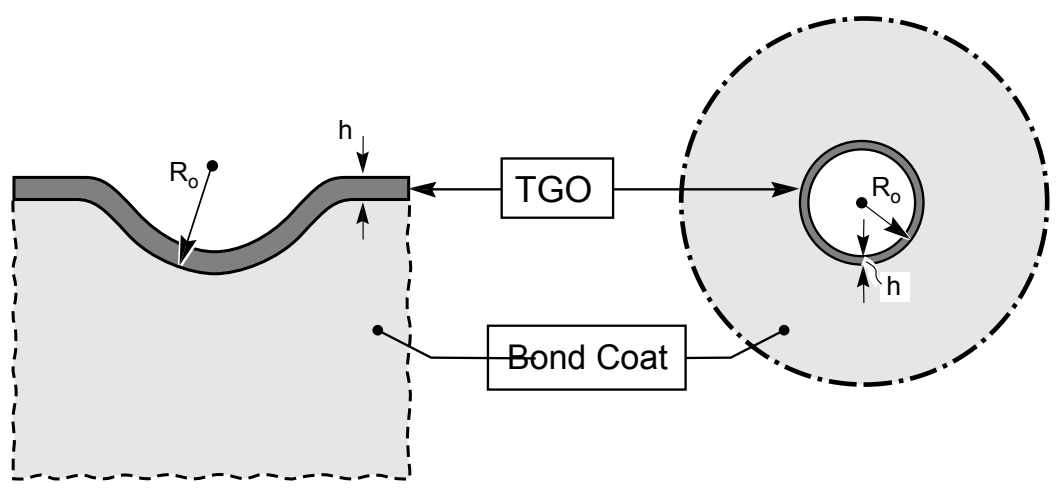

Fig. 5. A schematic of a hemispherical imperfection and of the spherical shell model.

\section{Oxidation Mechanism}

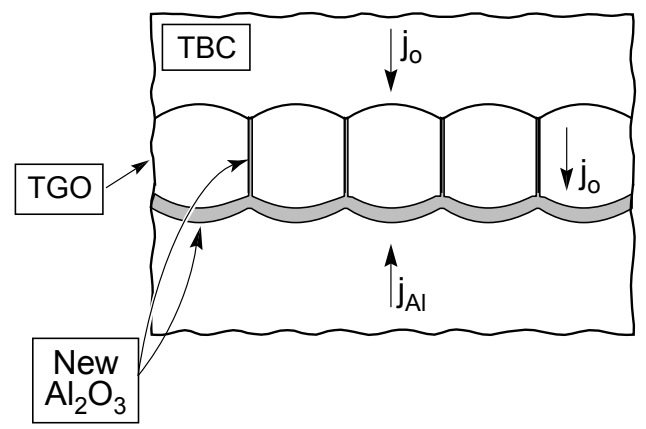

Fig. 6. Schematic indicating the matter deposition that causes thickening and lengthening of the TGO during oxidation (Evans et al., 2001). The latter results in a lateral growth strains, which motivate the instabilities depicted in Fig. 2.

growth that occur (a) at yield and (b) while it is elastic. A small contribution to the force on the bond coat arises because volume is conserved and the extra TGO is redistributed into thickening by plastic flow (Karlsson et al., 2002b). This redistribution results in a thickening per cycle:

$$
\Delta h=h\left(\Delta \varepsilon_{\mathrm{g}}-\Delta \varepsilon_{\mathrm{g}}^{\prime}\right)
$$


where $\Delta \varepsilon_{\mathrm{g}}^{\prime}$ is the incremental growth per cycle before yielding occurs $\left(0 \leqslant \Delta \varepsilon_{\mathrm{g}}^{\prime} \leqslant \Delta \varepsilon_{\mathrm{g}}\right)$. This effect is neglected in the following analytical model, but is included in the numerical simulation.

Finally, some semantics are clarified. The event depicted in Fig. 2 is termed an instability in the thermally grown oxide. Previously, for two reasons, it has been referred to as ratcheting. (a) It is often accompanied by cyclic yielding of the adjacent bond coat (He et al., 2000; Karlsson and Evans, 2001). (b) At higher levels of yield strength and at smaller imperfections it has characteristics indicative of shakedown. The present model demonstrates that, while cyclic yielding accelerates the process, the displacements occur without reversed plasticity. Such a response does not coincide with the conventional definition of ratcheting (Bree, 1968). Hereafter, the event is referred to as an instability of a form dominated by propagation, since substantial imperfections pre-exist that negate initiation requirements.

\section{Model definition}

The hemispherical undulation representative of actual imperfections (Figs. 2 and 3) is modeled by a fully spherical geometry (Fig. 5). The TGO is represented as a thin spherical shell of thickness $h$ and radius $R_{0}$ attached to an infinite matrix of bond coat (Fig. 5). Growth of the TGO shell radius is interpreted as undulation growth, since the plastic strains around imperfections are primarily radial in nature (He et al., 2000, 2002; Karlsson and Evans, 2001; Karlsson et al., 2002a, b). A comprehensive summary of the cyclic stresses and strains that arise in a related spherically symmetric model in the absence of a growth strain was developed by Olsson et al. (1995). In this analysis, no attempt is made to characterize the temperature-dependent creep behavior of the bond coat. Instead, it is modeled as elastic-perfectly plastic with temperature-independent yield strength $\sigma_{\mathrm{Y}}^{\mathrm{bc}}$. The temperature dependence of $\sigma_{\mathrm{Y}}^{\mathrm{bc}}$ affects the growth rate (Karlsson and Evans, 2001), but the phenomenon remains the same. To facilitate the analysis, the bond coat is taken to be elastically incompressible with Young's modulus, $E_{\mathrm{bc}}$. Except at the highest temperature in the history (the growth temperature $T_{0}$, Fig. 8), the TGO undergoes only elastic deformations governed by its Young's modulus, $E_{\text {tgo }}$, and Poisson's ratio, $v_{\text {tgo }}$. At $T_{0}$, the TGO is elastic-perfectly plastic with yield strength $\sigma_{\mathrm{Y}}^{\text {tgo }}{ }^{1}$ As already mentioned, TGO yielding plays a central role. Lateral (in-plane) growth of the TGO is emphasized, since this gives rise to the compressive stress that drives the increase in radius. The lateral growth strain over the entire thermal history is denoted by $\varepsilon_{\mathrm{g}}^{0}$. Two basic thermal histories are addressed (Fig. 7). (a) A single thermal cycle, wherein the full strain $\varepsilon_{\mathrm{g}}^{0}$ occurs while the system is at the growth temperature. (b) A multicycle scenario, with $N$ cycles, wherein the growth strain per cycle is $\Delta \varepsilon_{\mathrm{g}}=\varepsilon_{\mathrm{g}}^{0} / N$ (Fig. 7). Minor differences exist in the cycles chosen for the analytical and numerical models, as evident from Fig. 7. These differences do not

\footnotetext{
${ }^{1}$ This is achieved in the numerical model by letting $\sigma_{\mathrm{Y}}^{\text {tgo }}=10 \mathrm{GPa}$ for $T<900^{\circ} \mathrm{C}, \sigma_{\mathrm{Y}}^{\text {tgo }}=1 \mathrm{GPa}$ for $T>1000^{\circ} \mathrm{C}$, and letting $\sigma_{\mathrm{Y}}^{\text {tgo }}$ vary linearly in between.
} 

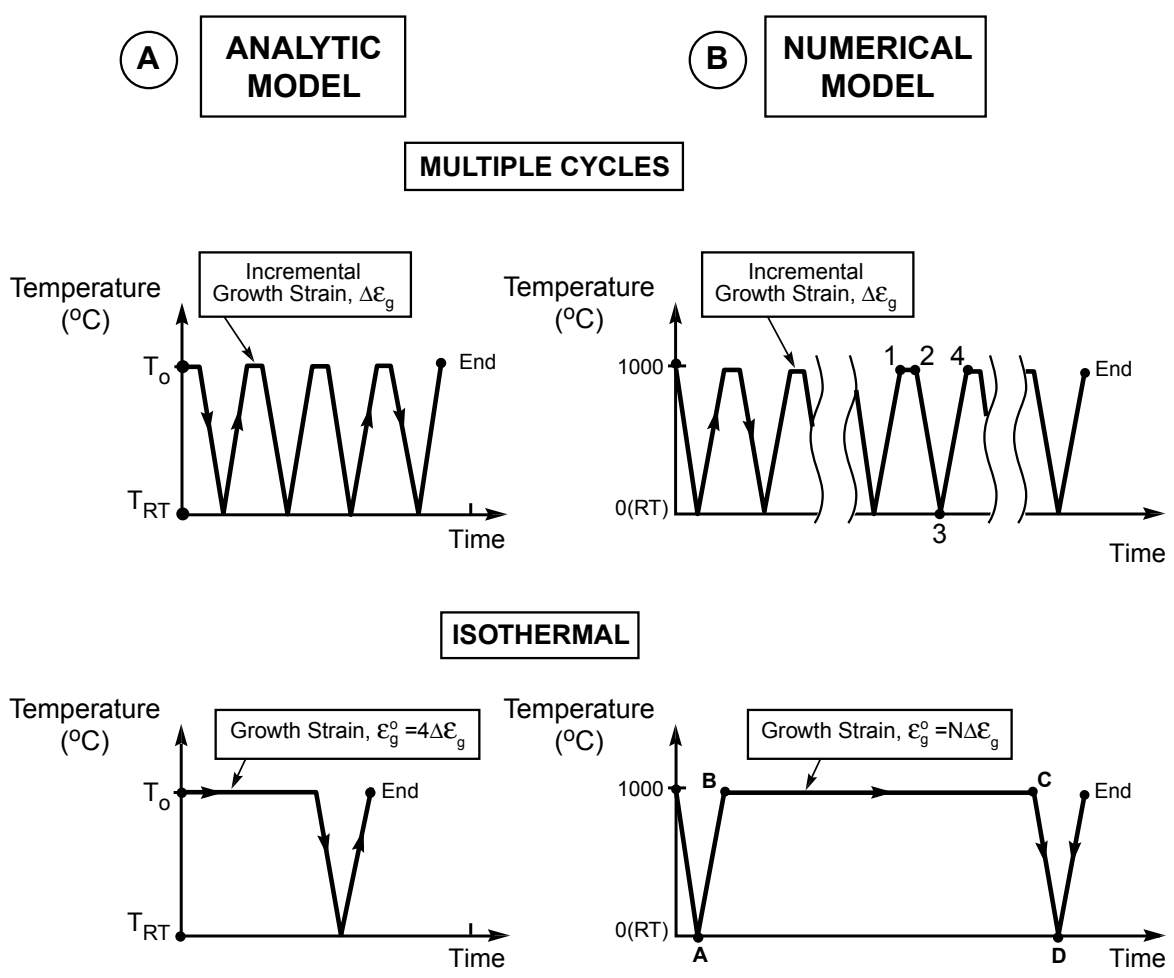

Fig. 7. The two thermal histories used to conduct calculations with the analytical and numerical models.

affect the basic phenomena. The cycle for the analytic model has been chosen to facilitate the presentation of results. That chosen for the numerical model is taken to be consistent with previous simulations (He et al., 2000, 2002; Karlsson and Evans, 2001; Karlsson et al., 2002a, b).

At the peak temperature, $T_{0}$, the system is driven by the increment of growth strain. During cooling to room temperature, $T_{\mathrm{RT}}$, and reheating to $T_{0}$, no growth strain occurs, and the system is driven by the thermal expansion misfit, $\Delta \alpha=\alpha_{\mathrm{bc}}-\alpha_{\mathrm{tgo}}(\Delta \alpha>0)$. The presentation of results is simplified considerably by the precise equivalence of increments of lateral growth strain, $\Delta \varepsilon_{\mathrm{g}}$, and increments in thermal expansion mismatch, $\Delta \alpha \Delta T$. Each is equivalent to a transformation strain, $\Delta \varepsilon_{\mathrm{T}}$, in the TGO, with due regard for sign.

The following additional notation is needed. The equi-biaxial stress in the TGO shell is $\sigma_{\mathrm{tgo}}$, the pressure between the shell and the matrix is $p \equiv-\sigma_{\mathrm{rr}}$, and the radius of the plastic zone in the bond coat is $R_{\mathrm{P}}$. For presentation of the results, it is convenient to let $u$ be the increase in the radius of the TGO shell, with the uniform expansion of the bond coat subtracted. That is, the actual change in the radius of the shell at any temperature $T$ would be: $u+\alpha_{\mathrm{bc}}\left(T-T_{0}\right) R$. 


\section{Analytical model}

\subsection{Basic relations}

Results are obtained by coupling the solution for a shell to the exact spherically symmetric solutions for an infinite matrix. Segments of the cyclic thermal history are defined by the occurrence of yielding in the bond coat and TGO. The yield condition for the bond coat under spherically symmetric conditions is $\left|\sigma_{\mathrm{rr}}-\sigma_{\theta \theta}\right|=\sigma_{\mathrm{Y}}^{\mathrm{bc}}$. For the TGO, the yield condition at the growth temperature is $\left|\sigma_{\text {tgo }}\right|=\sigma_{\mathrm{Y}}^{\text {tgo }}$. To assemble the complete thermal history, four possible combinations must be considered: (a) the bond coat and TGO both deform elastically; (b) both deform plastically; (c) the bond coat remains elastic while the TGO is plastic and (d) vice versa.

When the bond coat responds elastically the increments of pressure, TGO stress and relative displacement are given by

$$
\begin{aligned}
& \Delta p=\Delta \varepsilon_{\mathrm{T}}\left[\frac{3}{4 E_{\mathrm{bc}}}+\frac{R_{0}\left(1-v_{\mathrm{tgo}}\right)}{2 h E_{\mathrm{tgo}}}\right]^{-1}, \\
& \Delta \sigma_{\mathrm{tgo}}=-\frac{R_{0} \Delta p}{2 h} \\
& \frac{\Delta u}{R_{0}}=\frac{3 \Delta p}{4 E_{\mathrm{bc}}}
\end{aligned}
$$

The change in transformation strain, $\Delta \varepsilon_{\mathrm{T}}$, is either $-\Delta \alpha \Delta T$ or $\Delta \varepsilon_{\mathrm{g}}$, depending on the segment of the thermal history (cooling/reheating or growth, respectively). When the TGO is at yield, the situation changes, because the lateral growth exerts no pressure change: requiring that $\Delta \varepsilon_{\mathrm{T}}=0$ during that part of the growth segment.

Next, consider increments when a portion of the bond coat is at yield. The change in the stress in the bond coat at location, $r=R_{0}$, that affects its yielding response is

$$
\Delta \sigma_{\theta \theta}-\Delta \sigma_{\mathrm{rr}}=\frac{3}{2} \Delta p .
$$

In the thermal histories being considered, plastic yielding of the bond coat is found to occur during TGO growth and/or during cool-down. Reversed plasticity during heat-up, if it occurs, has only a small effect on the phenomenon of interest (as demonstrated in the following numerical assessment) and will be neglected: whereupon, plastic straining proceeds subject to radial compression with the yield condition, $\sigma_{\theta \theta}-\sigma_{\mathrm{rr}}=\sigma_{\mathrm{Y}}^{\text {bc }}$. The region undergoing plastic deformation is $R_{0} \leqslant r \leqslant R_{\mathrm{P}}$ (where $R_{\mathrm{P}}$ the radius of the plastic zone). Under these circumstances, the analysis simplifies, because $R_{\mathrm{P}}$ increases monotonically and can be directly connected to $p, \sigma_{\text {tgo }}$ and $u$, independent of the prior history, apart from knowledge of the accumulated transformation strain, $\varepsilon_{\mathrm{T}}$. Note that $\varepsilon_{\mathrm{T}}$ is the sum of all increments in $-\Delta \alpha \Delta T$ and $\Delta \varepsilon_{\mathrm{g}}$ when the TGO is not at yield. When the bond coat is at yield, the nonlinear equation relating $R_{\mathrm{P}}$ to the accumulated transformation strain, $\varepsilon_{\mathrm{T}}$, is

$$
\frac{1}{2}\left(\frac{R_{\mathrm{P}}}{R_{0}}\right)^{3}+\frac{\left(1-v_{\mathrm{tgo}}\right) E_{\mathrm{bc}} R_{0}}{E_{\mathrm{tgo}} h}\left(\frac{1}{3}+\ln \left(\frac{R_{\mathrm{P}}}{R_{0}}\right)\right)=\frac{\varepsilon_{\mathrm{T}}}{\varepsilon_{\mathrm{Y}}^{\mathrm{bc}}},
$$


where $\varepsilon_{\mathrm{Y}}^{\mathrm{bc}}=\sigma_{\mathrm{Y}}^{\mathrm{bc}} / E_{\mathrm{bc}}$. The corresponding results for $p, \sigma_{\mathrm{tgo}}$ and $u$ are

$$
\begin{aligned}
& \frac{\sigma_{\mathrm{tgo}}}{\sigma_{\mathrm{Y}}^{\mathrm{bc}}}=-\frac{R_{0}}{h} \frac{1}{3}+\ln \frac{R_{\mathrm{P}}}{R_{0}}, \\
& p=-\frac{2 h \sigma_{\mathrm{tgo}}}{R_{0}}, \\
& \frac{u}{R_{0}}=\frac{\varepsilon_{\mathrm{Y}}^{\mathrm{bc}}}{2} \frac{R_{\mathrm{P}}}{R_{0}} .
\end{aligned}
$$

For purposes of presenting the numerical results in Section 3.2, (5) can be rewritten in the non-dimensional form:

$$
\begin{aligned}
\frac{u}{R_{0} \varepsilon_{\mathrm{Y}}^{\mathrm{bc}}} & =\frac{\varepsilon_{\mathrm{T}}}{\varepsilon_{\mathrm{Y}}^{\mathrm{bc}}}-\Lambda \frac{E_{\mathrm{bc}} R_{0}\left(1-v_{\mathrm{tgo}}\right)}{E_{\mathrm{tg} 0} h}, \\
\frac{\sigma_{\mathrm{tgo}} h}{\sigma_{\mathrm{Y}}^{\mathrm{bc}} R_{0}} & =-\Lambda,
\end{aligned}
$$

where $\Lambda \equiv \frac{1}{3}+\ln \left(R_{\mathrm{P}} / R_{0}\right)$ is a slowly varying function of $\varepsilon_{\mathrm{T}} / \varepsilon_{\mathrm{Y}}^{\mathrm{bc}}$. Note that $\sigma_{\mathrm{Y}}^{\text {tgo }}$ does not enter (6) explicitly. It enters implicitly through its effect on $\varepsilon_{\mathrm{T}}$, as explained below.

As already noted, two basic problems are analyzed. In one, the system is subjected to a single cycle and in the other, to multiple thermal cycles, for identical total TGO growth (Fig. 7). It will be demonstrated that yield of the TGO plays an essential role in the difference between the responses found in these scenarios. For both examples, the following numerical values are used for the material parameters, temperature range and normalized TGO thickness:

$$
\begin{aligned}
E_{\mathrm{bc}} & =190 \mathrm{GPa}, \quad E_{\mathrm{tgo}}=380 \mathrm{GPa}, \quad v_{\mathrm{tgo}}=0.2, \\
\sigma_{\mathrm{Y}}^{\mathrm{bc}} & =200 \mathrm{MPa}, \quad \sigma_{\mathrm{Y}}^{\mathrm{tgo}}=1 \mathrm{GPa}\left(T=T_{0}\right), \\
\alpha & =6 \times 10^{-6} / C, \quad T_{0}-T_{\mathrm{RT}}=1000^{\circ} \mathrm{C}, \quad h / R_{0}=0.1 .
\end{aligned}
$$

To simplify the presentation of results, the thickening of the TGO that occurs while it is yielding (1) is ignored.

\subsection{Single cycle response}

The cycle being considered (Fig. 7) begins with a stress-free system at the growth temperature $T_{0}$. There are five stages. Three occur at $T=T_{0}$ as the TGO grows.

(i) Initially the bond coat responds elastically to the lateral growth strain in the TGO and (2) applies, until the bond coat begins to yield, as ascertained from (3).

(ii) Under continuing TGO growth, Eqs. (4) and (5) are operative with the bond coat at yield until the TGO yields (when $\sigma_{\text {tgo }}$ attains $-\sigma_{\mathrm{Y}}^{\text {tgo }}$ ).

(iii) Once the TGO yields, $\varepsilon_{\mathrm{T}}$ does not change, even though TGO growth continues, whereupon $R_{\mathrm{P}}, p, \sigma_{\mathrm{tpo}}, u$ remain constant. 
The fourth and fifth stages occur during cool down and reheating.

(iv) The TGO responds elastically and its compressive stress can exceed $\sigma_{\mathrm{Y}}^{\text {tgo }}$ (since $T<T_{0}$ ). The bond coat continues to deform plastically, with (4) and (5) in force and with $\varepsilon_{\mathrm{T}}$ increasing by $-\alpha T$. The maximum extent of the plastic zone and the maximum compressive stress in the TGO occur at the minimum temperature.

(v) During heat-up, the bond coat is assumed to respond elastically (reversed yield does occur, but it is neglected) and the variable changes are governed by (2) and (3).

Specific results for 4 cycles are presented in Fig. 8, computed using (7). The horizontal axis is numbered according to the five distinct steps in the deformation history. Yield of the TGO in the first cycle occurs when $\varepsilon_{\mathrm{g}}=0.0029$. Additional lateral growth strain in the TGO in this cycle produces no further change in any of the model variables. Thus, the plots of $\sigma_{\text {tgo }}$ and $u$ in Fig. 8 for the first cycle are equally applicable if the entire lateral growth strain, $\varepsilon_{\mathrm{g}}^{0}=0.02$, had occurred in the first cycle. Accordingly, the stresses and displacements during cooling are found by inserting $\varepsilon_{\mathrm{T}}=0.0029+\alpha\left(T_{0}-T\right)$ into (4) and (5), where $\alpha$ is given by (7) and $T$ is the current temperature.

\subsection{Multiple thermal cycles}

The preceding single cycle response is contrasted with that for a 4-cycle history (Fig. 8) for the same total growth strain, $\varepsilon_{\mathrm{g}}^{0}=0.02$, such that the growth strain per cycle is, $\varepsilon_{\mathrm{g}}=0.005$. The behavior in the first cycle is identical to that described above with yielding of the TGO beginning when $\varepsilon_{\mathrm{g}}=0.0029$. The steps in subsequent cycles differ. To aid interpretation, those steps in the history when the bond coat is at yield are highlighted as thinner lines in the figure. In the first step of the subsequent cycles, at $T=T_{0}$, the TGO growth strain increases and the bond coat responds elastically. In the second step, the TGO yields and grows without changing the stresses and displacements. As cool-down occurs, in the third step, the bond coat initially responds elastically, but then reaches yield. In the fourth step, during the remainder of cool-down, the bond coat is at yield throughout. The additional irreversible expansion of the TGO shell into the matrix occurs in this step. In the fifth and final step in the cycle, the bond coat responds elastically during reheating to $T_{0}$. Note that, even though the growth of the TGO occurs while the bond coat is elastic, the growth increases the stress in the matrix and in the TGO (prior to yielding) and sets the stage for a significant additional increase in the TGO radius during cool-down.

The expansion of the TGO radius, as measured by $u$ at the end of the fourth cycle, is many times its value at the end of the first cycle. Recall that $u$ is defined as the displacement of the TGO shell minus the uniform thermal expansion of the matrix referenced to the growth temperature: namely, $u$ is the increase of the radius of the TGO at $T_{0}$. After the first cycle, with $\varepsilon_{\mathrm{g}}=0.005, u$ is identical to the expansion that occurs when the full growth strain, $\varepsilon_{\mathrm{g}}^{0}=0.02$, occurs in a single cycle. This is the origin of the difference between the isothermal and cyclic response, as elaborated 


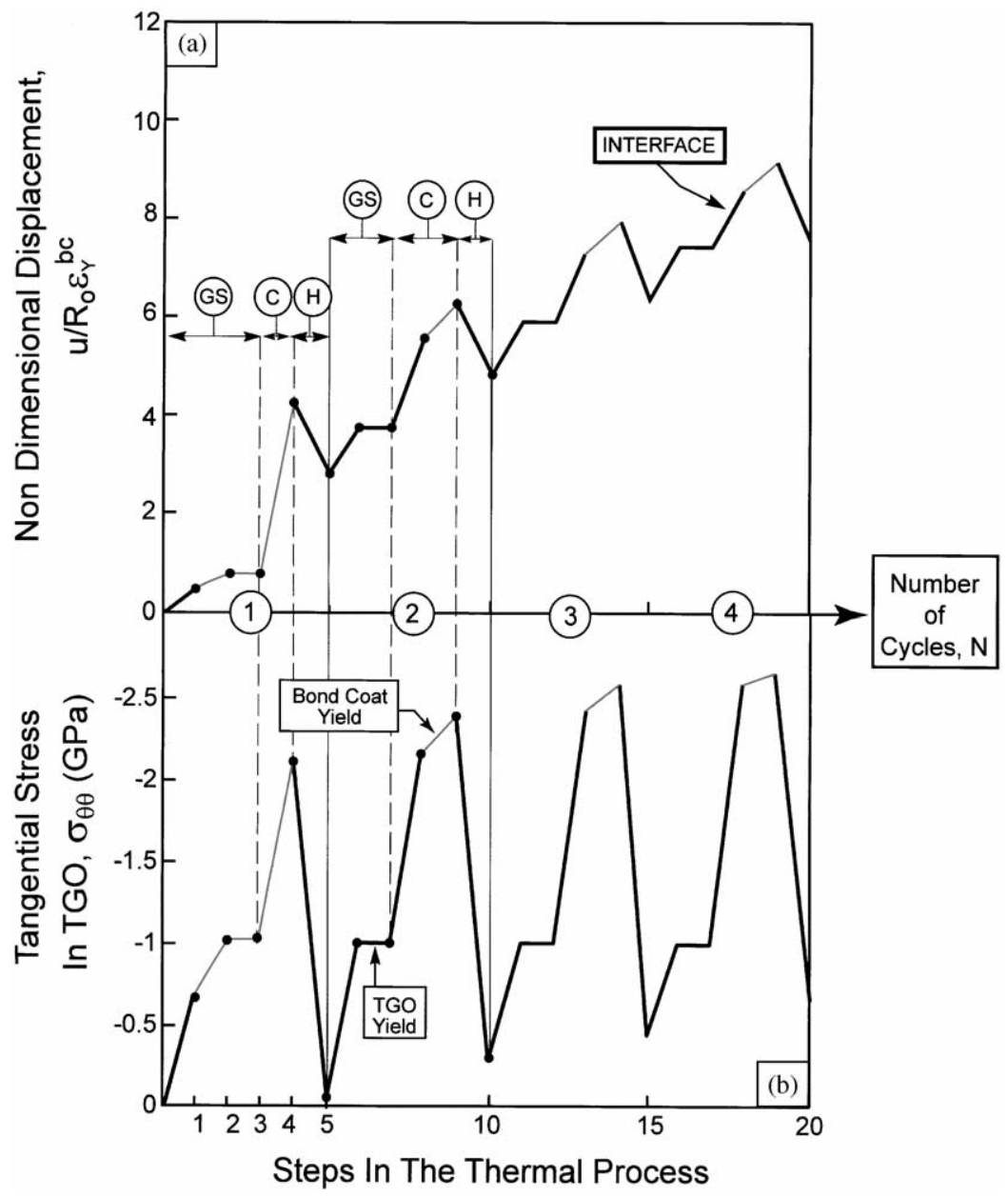

Fig. 8. A summary of the analytical results obtained using the parameters from (7). (a) Changes in the displacement of the TGO shell upon cycling, indicating the five stages defined in the text. (b) Changes in the tangential compression in the TGO. The thinner lines demark the stages wherein the bond coat is at yield. Otherwise, the bond coat responds elastically. Note that the response within the first cycle is equally applicable for both of the thermal histories indicated on Fig. 7, since the TGO yields once the growth strain reaches 0.0029 . Notations: GS - Growth stress: $\mathrm{C}$ - cooling, $\mathrm{H}$ - heating.

below. Note that, if the thermal history had involved more than four cycles, with the same total growth strain, $\varepsilon_{\mathrm{g}}^{0}=0.02$, the expansion of the TGO at the end of the history would have been even larger.

\subsection{The role of $T G O$ yield in the cyclic expansion}

In the preceding scenarios, had the TGO remained elastic, there would have been no difference between the expansion of the TGO under single and multiple cycle 
histories. This assertion, which is verified by the following numerical calculations (Section 4), can be appreciated by referring to (4) and (5) with the aid of the following argument. If the TGO responds purely elastically during growth strain, the net transformation strain just before the end of the single cycle, prior to heat-up (when $T=T_{\mathrm{RT}}$ ), is $\varepsilon_{\mathrm{T}}=\varepsilon_{\mathrm{g}}^{0}-\alpha\left(T_{0}-T_{\mathrm{RT}}\right)$. The radius of the plastic zone and the magnitudes of the other variables are given by inserting this $\varepsilon_{\mathrm{T}}$ into (4) and (5). For the cyclic thermal history, when $T=T_{\mathrm{RT}}$, the net transformation strain is again $\varepsilon_{\mathrm{T}}=\varepsilon_{\mathrm{g}}^{0}-\alpha\left(T_{0}-T_{\mathrm{RT}}\right)$, provided that the TGO does not yield. The last step, heating-up to $T_{0}$, involves only elastic deformations and is the same for the two histories.

To summarize, yielding of the TGO at the growth temperature accommodates the growth strain without incremental expansion of the radius of the TGO shell. When growth occurs in a single thermal cycle, most of that growth takes place with the TGO at yield. Conversely, when the same growth is spread over multiple thermal cycles, a greater fraction of growth takes place when the TGO is elastic, and the effect of growth on the expansion of the TGO shell is greater. As seen from the example, the difference in expansion between the two histories can be substantial.

\section{Numerical simulations}

The finite element code ABAQUS has been used to perform spherical shell simulations (Fig. 5), utilizing large deformation theory. The goals are to affirm the analytical results and to address the consequences of the simplifications. Initially, the same properties (7) are used and later varied in a sensitivity study. Growth of the TGO is simulated by imposing stress-free strains in accordance with a user subroutine, uexpan (Karlsson and Evans, 2001). The in-plane strain, $\varepsilon_{\mathrm{g}}$, is imposed uniformly through the TGO-thickness. Thickening of the TGO by growth is neglected (but it does thicken in accordance with (1)), except in the sensitivity analysis. To be consistent with prior analyses (He et al., 2000, 2002; Karlsson and Evans, 2001; Karlsson et al., 2002a, b), all calculations start with a cooling-heating sequence before the growth strain is applied (Fig. 7).

\subsection{Comparisons with analytic results}

Numerical results amenable to direct comparison with the preceding analytical results (Fig. 8) are presented in Fig. 9. The numerical results are presented in terms of cycles rather than growth steps. Nevertheless, the same sequence of steps is still apparent. Moreover, there is an almost exact overlap of the numerical and analytical predictions: both the displacements (Figs. 8a and 9a) and the tangential stresses in the TGO (Figs. $8 \mathrm{~b}$ and $9 \mathrm{~b})$ are essentially identical. ${ }^{2}$ Note that the simulation predicts reverse yielding

\footnotetext{
2 The slopes of the individual segments are different in the two figures. This is because the abscissa for the analytical model (Fig. 8) is presented with respect to steps in the thermal cycle, while the abscissa for the numerical model relates to the number of cycles.
} 


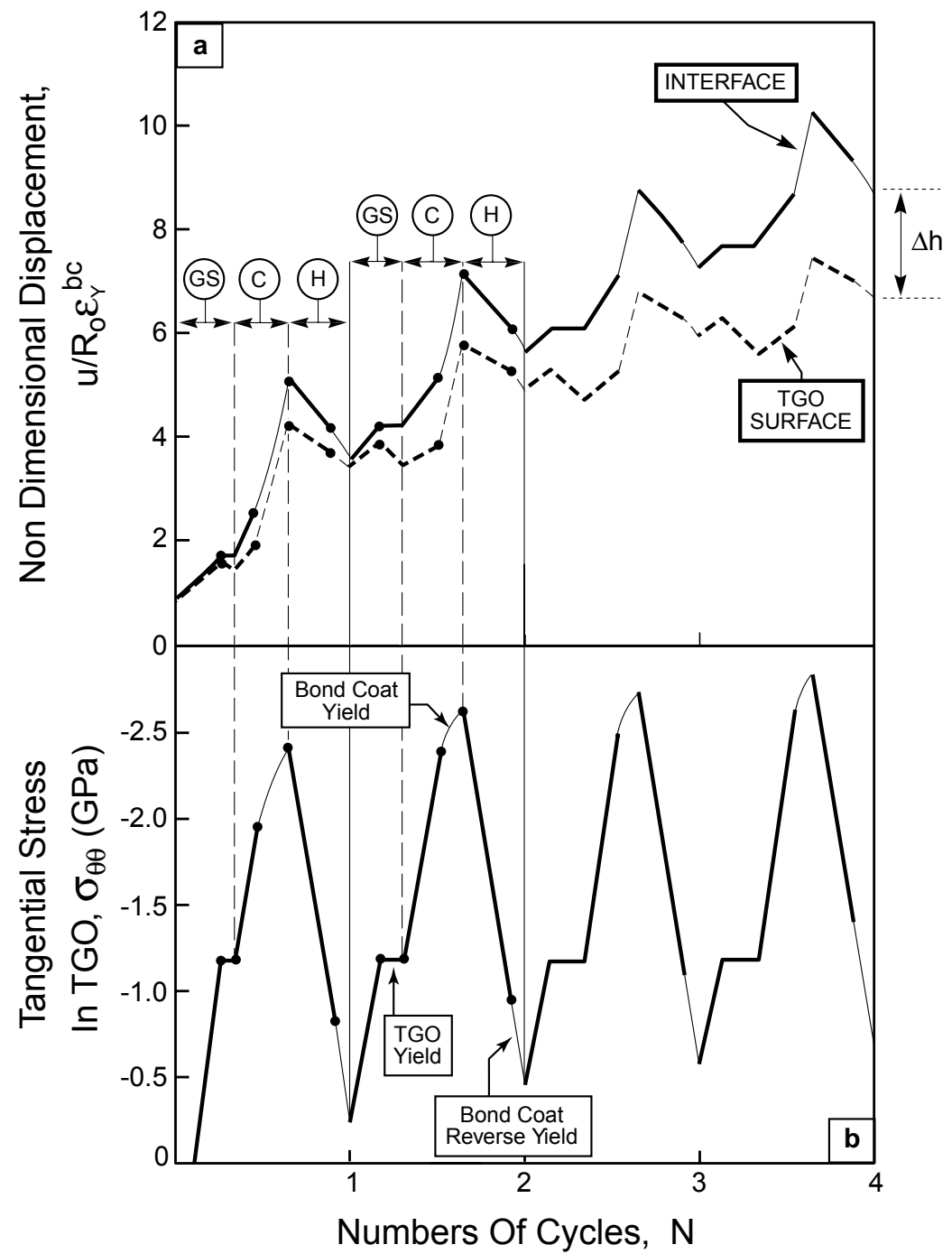

Fig. 9. A summary of the numerical simulations conducted for the same parameters used to construct Fig. 8: (a) displacements and (b) stresses. The thinner lines demark the stages wherein the bond coat is at yield. Otherwise, the bond coat responds elastically. The locations of the TGO surface and the interface with the bond coat have both been plotted on (a) to reveal the changes in TGO thickness, $h$, as the system cycles. The preceding half cycle cooling-heating (used in the numerical simulations but not in the analytical model, see Fig. 7) is not shown, for direct comparison with Fig. 8. Notations: GS-Growth stress: $\mathrm{C}$-cooling, $\mathrm{H}$-heating.

during reheating, as apparent from a plot of the development of stresses and strains in the bond coat (see Fig. 12): an effect neglected in the analytical model. Yet, this neglect appears not to have adversely influenced the good correspondence between the numerical and analytical results. Accordingly, it may be concluded that reverse yielding 


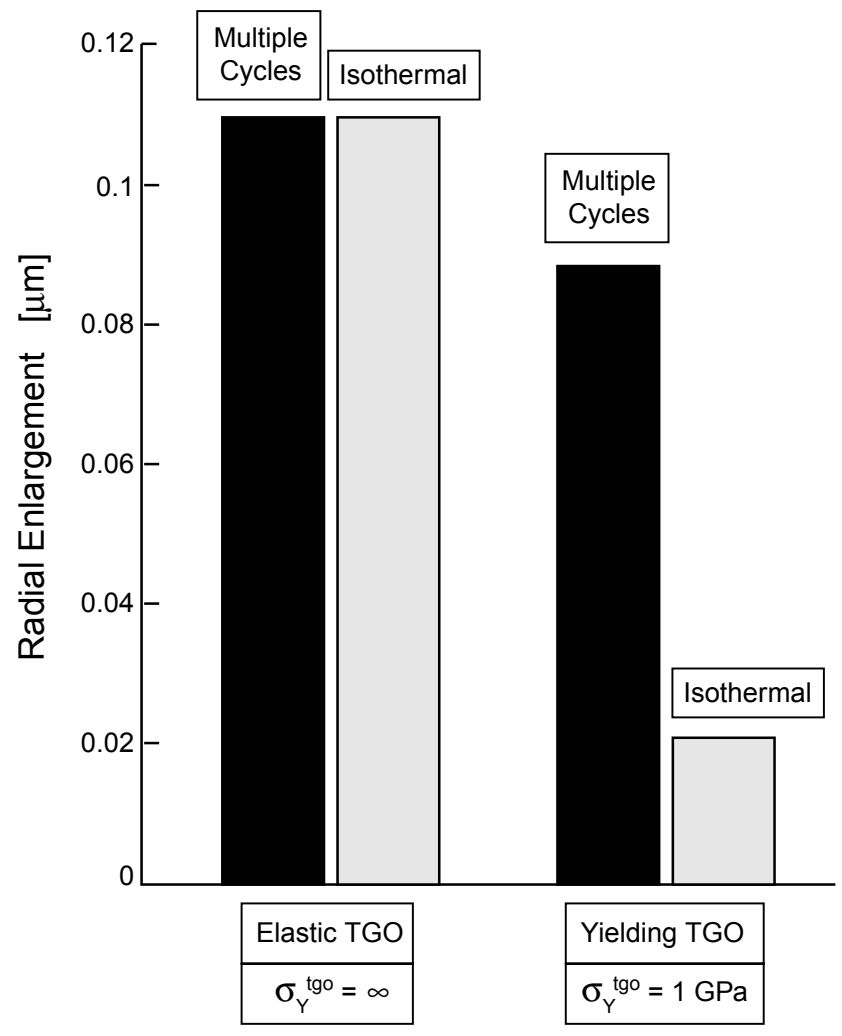

Fig. 10. Simulations comparing the radial enlargement for isothermal and cyclic conditions, for an elastic TGO $\left(\sigma_{\mathrm{Y}}^{\text {tgo }}=\infty\right)$ and a yielding TGO $\left(\sigma_{\mathrm{Y}}^{\text {tgo }}=1 \mathrm{GPa}\right), \sigma_{\mathrm{Y}}^{\mathrm{bc}}=200 \mathrm{MPa}, N=24, \quad \varepsilon_{\mathrm{g}}=10^{-3}$. Absent yielding, the same radial change occurs for isothermal and cyclic conditions.

has a minor effect on the system response. The situation will be discussed further in connection with the sensitivity analysis. Note that the TGO yields when the tangential TGO stress is about $-1.2 \mathrm{GPa}$, even though $\sigma_{\mathrm{Y}}^{\text {tgo }}=1 \mathrm{GPa}$. This is due to the tri-axial state of stress, which is neglected in the analytical model.

The location of the TGO surface is also plotted in Fig. 9a and the difference between the interface and surface curves corresponds to a thickness change in the TGO. A permanent increase in TGO thickness commences when the TGO yields, as elaborated below. At room temperature, the thickness change in the TGO at ambient, relative to that at the elevated temperature, is also due to the thermal contraction. Both these effects have been subtracted in the analytical model.

The analytical model implies that, when the TGO does not yield, the change in the shell radius, $u$, is the same for multiple and single cycle histories, but differs when the TGO yields. This implication is affirmed by the simulations summarized in Fig. 10. Note that, when $\sigma_{\mathrm{Y}}^{\text {tgo }}=1 \mathrm{GPa}, \quad u$ after 24 cycles is almost five times larger than after a single cycle. The effect is rationalized upon comparing the radial 


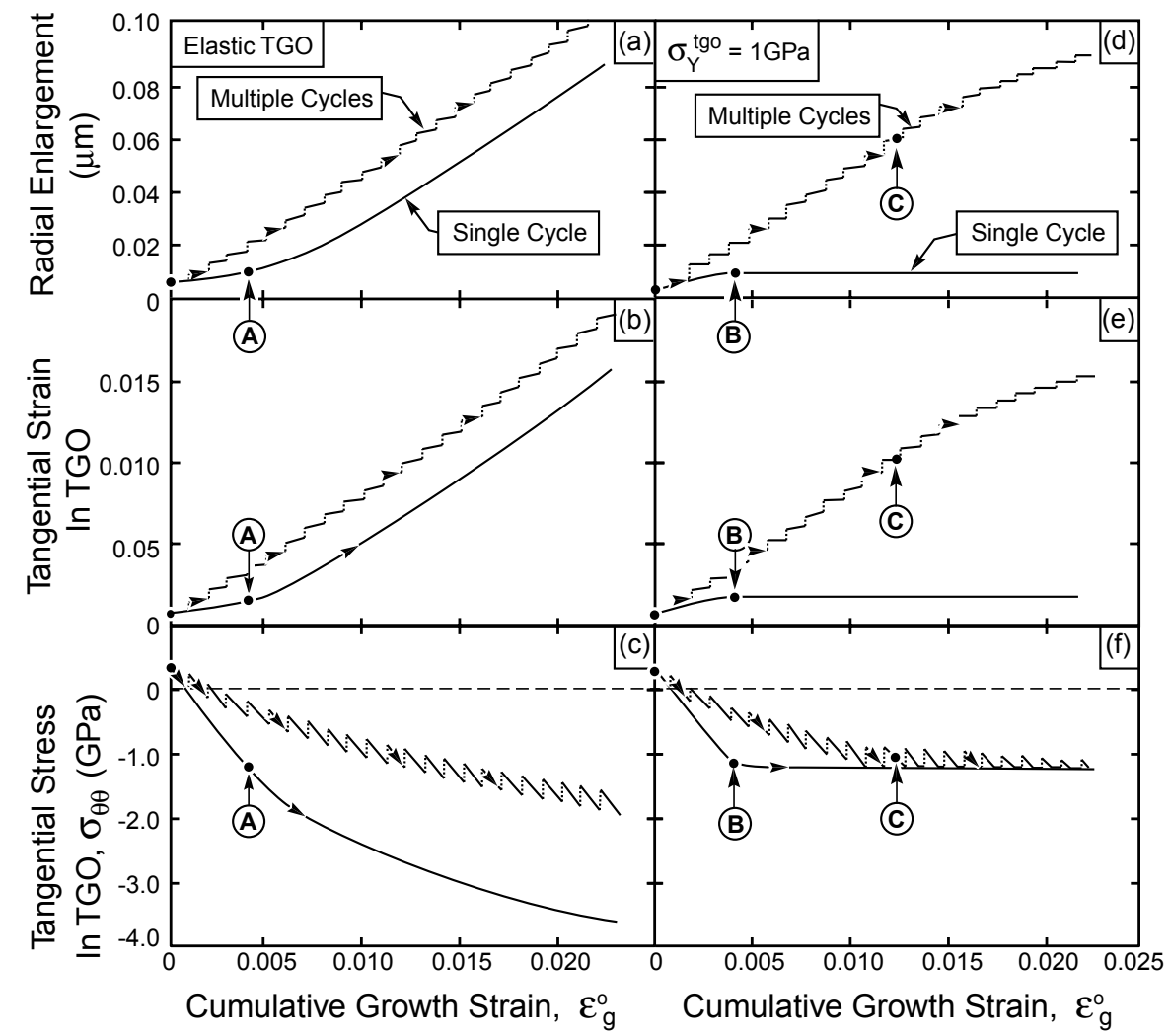

Fig. 11. A comparison of responses for cyclic and isothermal conditions for an elastic TGO $\left(\sigma_{\mathrm{Y}}^{\text {tgo }}=\infty\right)$ and a yielding TGO $\left(\sigma_{\mathrm{Y}}^{\text {tgo }}=1 \mathrm{GPa}\right)$, as a function of the cumulative growth strain: $(\mathrm{a}, \mathrm{d})$ radial enlargement, (b,e) tangential strain in TGO, $(\mathrm{c}, \mathrm{f})$ tangential stress in TGO. $\left(\sigma_{\mathrm{Y}}^{\mathrm{bc}}=200 \mathrm{MPa}\right)$. The position $\mathbf{A}$ refers to incipience of yielding in the bond coat for elastic TGO. For the case of yielding in the TGO, the positions designated $\mathbf{B}$ refer to the onset of TGO yielding for single cycle simulations, while positions $\mathbf{C}$ refer to TGO yielding in multicycle simulations. Note that, in the isothermal scenario, the changes in displacement and stress stop once the TGO yields. Under cyclic conditions, displacements and stresses continue to change upon TGO yielding, albeit at a lower rate. In this scenario, $\mathbf{C}$ designates the transition from "transient" to "steady-state" responses.

expansion, the tangential stress in the TGO, $\sigma_{\theta \theta}^{\text {tgo }}$, and the tangential strain in the TGO, $\varepsilon_{\theta \theta}^{\text {tgo }}$ for an elastic TGO and for a TGO that yields, with $\sigma_{\mathrm{Y}}^{\text {tgo }}=1 \mathrm{GPa}$ (Fig. 11). For the elastic TGO (Figs. 11A-C), after a settling-in stage, the strains and displacements increase with increase in growth strain at precisely the same rate for isothermal and cyclic conditions. Also, the stress in the TGO continues to increase, quickly attaining levels $(\rightarrow 4 \mathrm{GPa})$ that cannot be sustained without yielding or creep. Introducing TGO yielding causes dramatic changes in response (Figs. 11D-F). For isothermal conditions, the displacements and strains per growth strain increment, $\varepsilon_{\mathrm{g}}$, decrease dramatically once the TGO yields. For the corresponding cyclic simulation, the stresses in the TGO remain elastic. This difference arises because the plasticity in the bond 


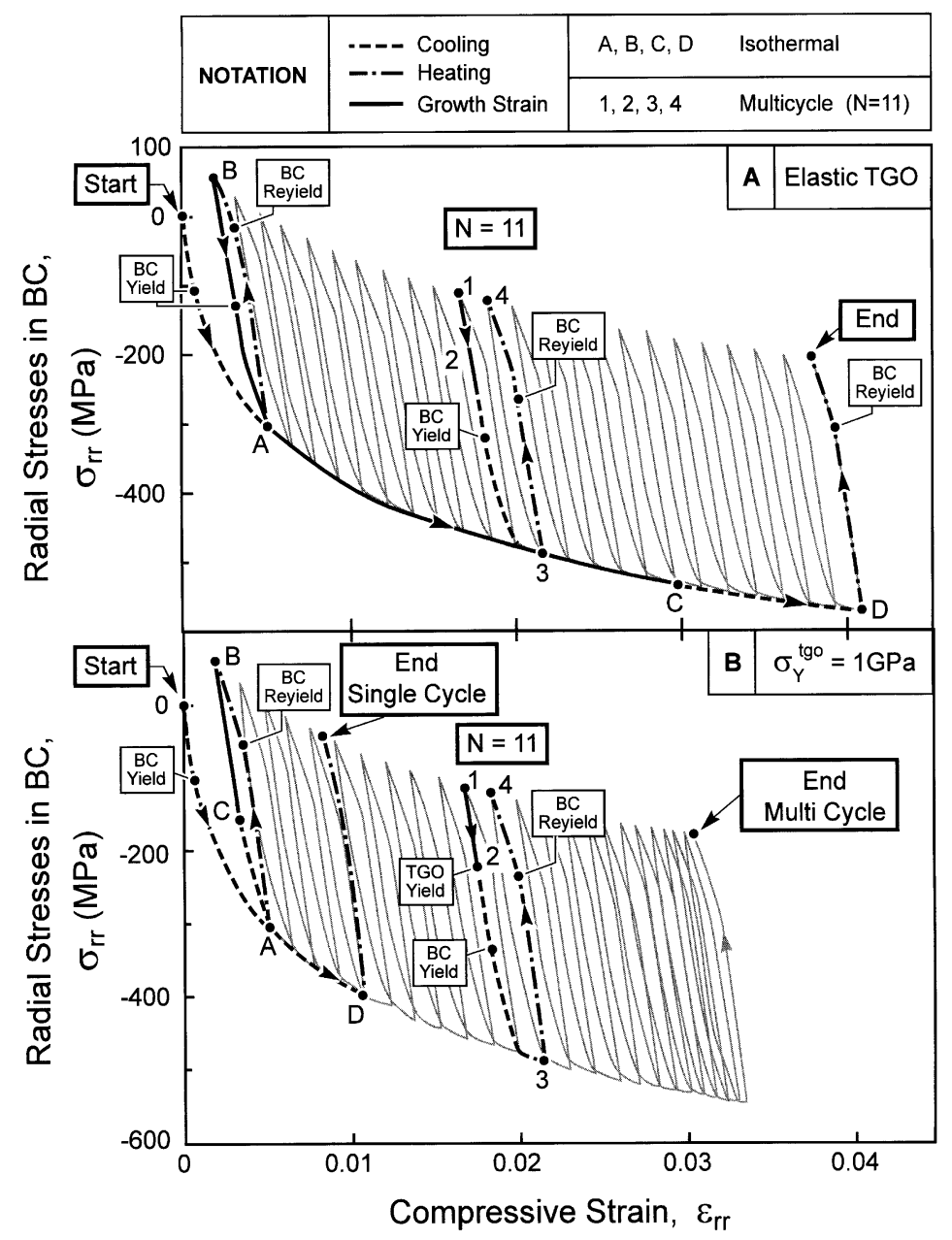

Fig. 12. The relationship between the radial stress and strain in the bond coat next to the TGO for single and multicycle conditions $\left(\sigma_{\mathrm{Y}}^{\mathrm{bc}}=200 \mathrm{MPa}\right)$ : (a) elastic TGO, (b) yielding TGO, $\sigma_{\mathrm{Y}}^{\text {tgo }}=1 \mathrm{GPa}$. For the multicycle simulations, the response in the 11th cycle is highlighted for clarity. The locations 1 through 4 coincide with those indicated on Fig. 7. For the isothermal simulations, the locations A through D are those presented on Fig. 7. For the elastic TGO, note that the end point is exactly the same for the two thermal histories. Conversely, when the TGO yields, the end point for the cyclic simulation occurs at a much larger strain level than that for the isothermal simulation.

coat on cooling diminishes the tangential stress induced in the TGO upon reheating to the growth temperature, enabling $\varepsilon_{\mathrm{g}}$ to be added without reaching $\sigma_{\mathrm{Y}}^{\text {tgo }}$. This large difference between the cyclic and isothermal responses continues until the stress in the TGO becomes sufficiently compressive on reheating that the strain addition $\varepsilon_{\mathrm{g}}$ causes yielding. This occurrence constitutes the transition to "steady-state" discussed above.

The associated changes in the radial stresses and strains in the bond coat ( $\sigma_{\mathrm{rr}}^{\mathrm{bc}}$ and $\varepsilon_{\mathrm{rr}}^{\mathrm{bc}}$, respectively) next to the TGO are compared in Fig. 12. For the elastic TGO, the 


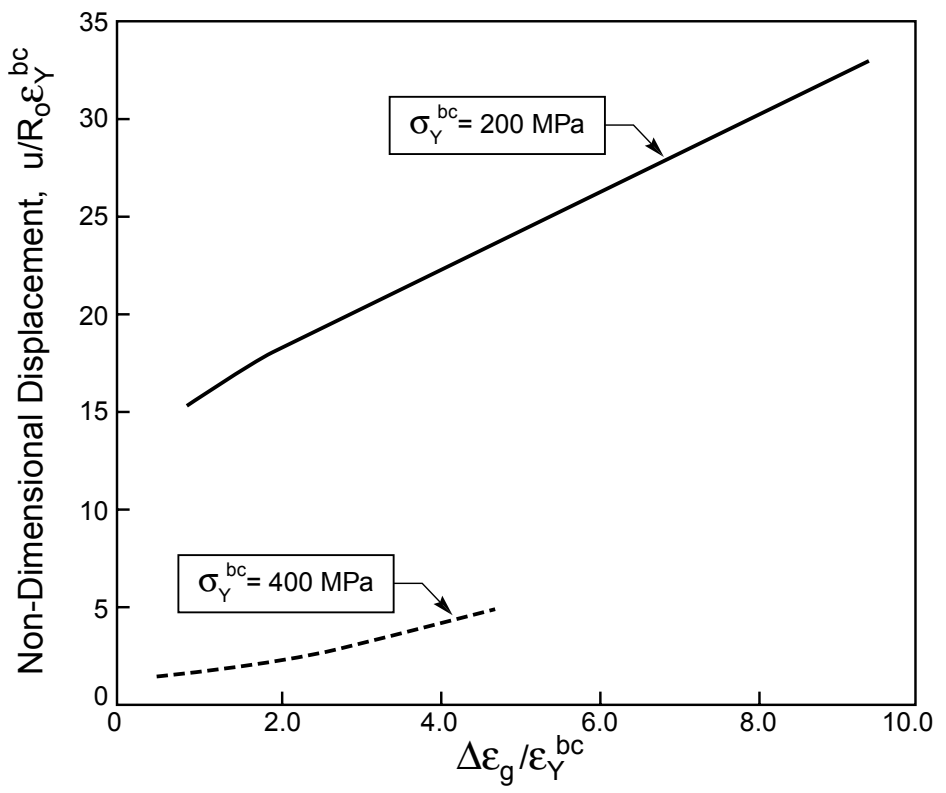

Fig. 13. Numerical simulations showing that the radial enlargement increases with increasing incremental growth strain (after 24 cycles, $\sigma_{\mathrm{Y}}^{\text {tgo }}=1 \mathrm{GPa}$ ).

final state of radial stress and strain is the same for isothermal and cyclic conditions, with large strains induced in the bond coat for both conditions. When the TGO is allowed to yield, there is a large difference, with substantially smaller strains induced under isothermal conditions, consistent with the discussion in Section 2.5. Since the changes in $\sigma_{\mathrm{rr}}^{\mathrm{bc}}\left(\varepsilon_{\mathrm{rr}}^{\mathrm{bc}}\right)$ are intricate, clarification of Fig. 12 is provided in the appendix, where it will be shown that reverse yielding occurs upon reheating.

\subsection{Sensitivity study}

In order to affirm the sensitivity of the instability to material properties predicted by the analytic solutions, and to explore several additional effects, simulations have been conducted for a wider range of yield strengths, $\sigma_{\mathrm{Y}}^{\text {tgo }}$, and $\sigma_{\mathrm{Y}}^{\mathrm{bc}}$, and plotting the results in accordance with the non-dimensional coordinates suggested by (6) (Figs. 13-16). Simulations of the radial expansion (Fig. 13) reveal that it does not increase with increasing growth strain in the strictly linear manner predicted analytically (2). Deviations occur once the TGO yields and are more pronounced at the higher bond coat yield strength $\left(\sigma_{\mathrm{Y}}^{\mathrm{bc}}=400 \mathrm{GPa}\right)$ and at larger growth strains. This discrepancy arises because the TGO thickens once it yields (1), resulting in a larger pressure,

$p$, despite the constant stress in the TGO. This effect was ignored in the analytical model. 


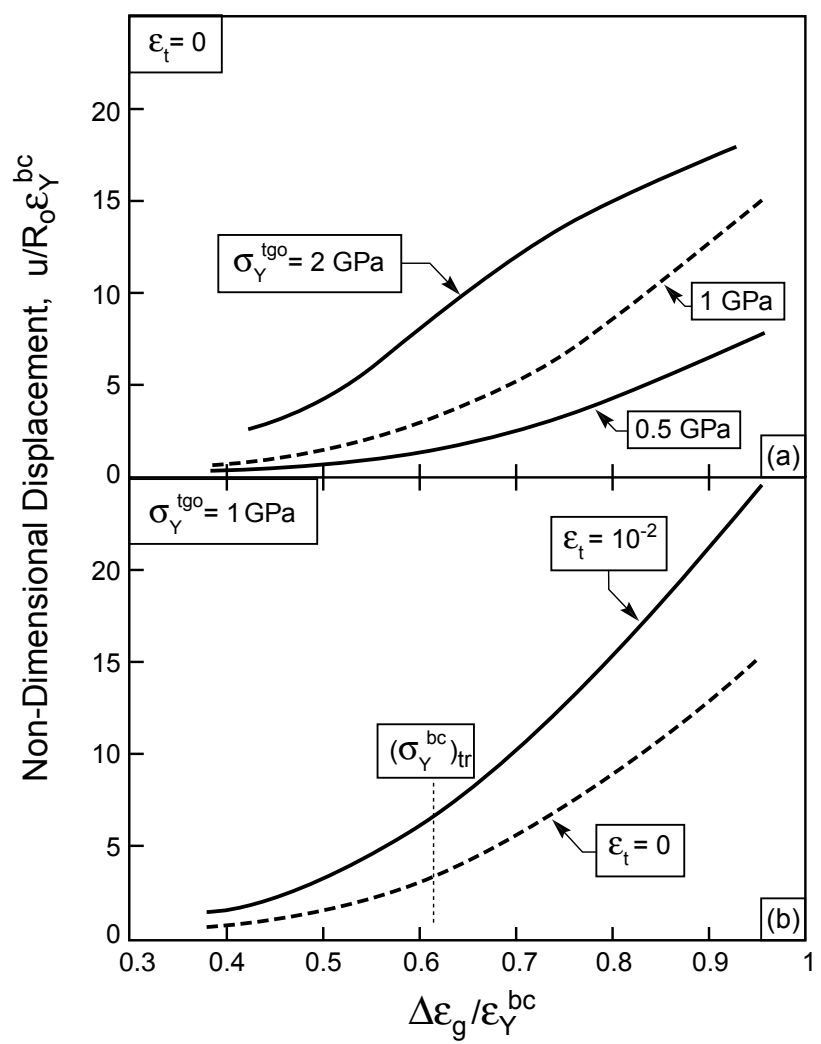

Fig. 14. The non-dimensional radial enlargement $(N=24)$ as an inverse function of bond coat yield strain (for growth strain $\varepsilon_{\mathrm{g}}=10^{-3}$ ). (a) Larger TGO yield strength results in smaller displacements. (b) Including a thickening growth strain leads to larger displacements but similar trends.

The influence of TGO yield strain for various bond coat yield strengths, at $N=24$, is presented on Fig. 14a and the relative role of the thickening strain on Fig. 14b. The bond coat yield strength is normalized according to (6). The thickening strain increases the displacement, but does not change the general behavior. The slope, $\mathrm{d} u / \mathrm{d} N$, increases abruptly at a "transition" yield strength $\left[\left(\sigma_{\mathrm{Y}}^{\mathrm{bc}}\right)_{\operatorname{tr}} \approx 300 \mathrm{MPa}\right.$ for $\left.\varepsilon_{\mathrm{t}}=0\right]$. This response led to the earlier notion of a transition from shakedown to ratcheting, associated with the onset of reverse yielding (He et al., 2002; Karlsson and Evans, 2001). Now, predicated on the preceding arguments, it is apparent that reverse yielding does not play a fundamental role. It simply signifies a change from a slow to a rapid displacement-rate.

The appreciable increase in displacement-rate upon increasing the TGO yield strength, $\sigma_{\mathrm{Y}}^{\text {tgo }}$ (in the range $0.5,1,2 \mathrm{GPa}$ ) (Figs. 15 and 16) distinguishes the transition from transient to steady-state, which commences at $N_{\text {tr }}$ cycles. Note that the transient stage, $N \leqslant N_{\text {tr }}$, enlarges as the TGO yield strength increases, with a small effect of 


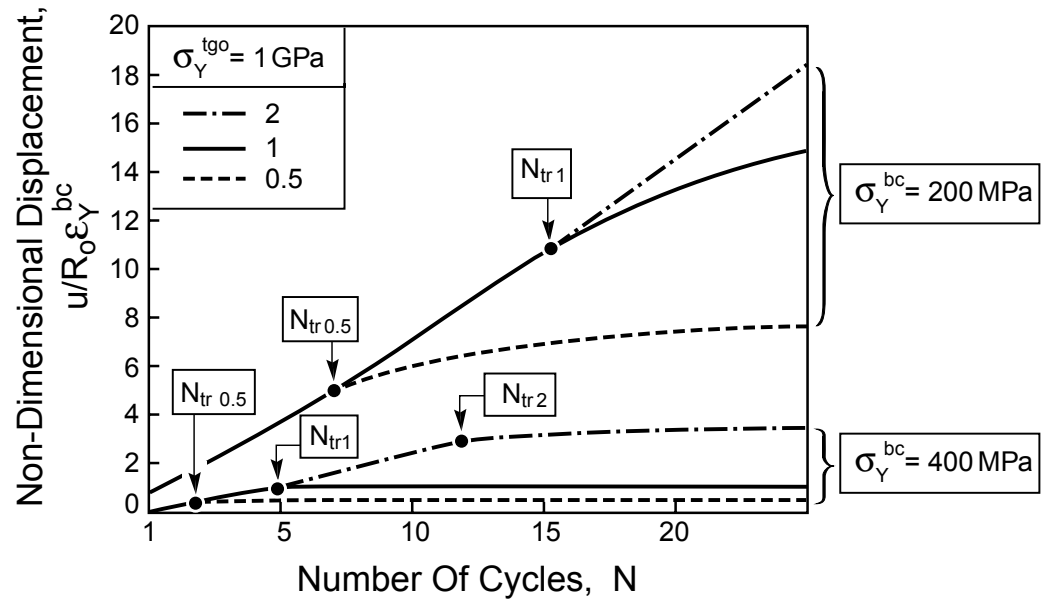

Fig. 15. The cycle-by-cycle development of the non-dimensional radial enlargement for various TGO yield strengths. When TGO yielding occurs, $N_{\mathrm{cr}}$ a steady-state is reached wherein the enlargement is caused entirely by the thickening of the TGO.

the bond coat yield strength. Furthermore, the transition coincides with a thickening of the TGO per cycle (Fig. 9), once TGO yielding commences, attributed to material redistribution described above (Karlsson and Evans, 2001).

\section{Concluding remarks}

A spherically symmetry model is devised to facilitate understanding of the propagation of morphological instabilities observed in a class of thermal barrier system. A thin shell represents the TGO. The surrounding medium is the bond coat. In the model, the expansion of the TGO is monitored as the system is subjected to various thermal cycling histories. An analytical model with some simplifications is used to establish the salient non-dimensional parameters and to provide basic insights. A numerical simulation, conducted with finite elements, is used to affirm the main implications from the analytical model and to explore additional characteristics. The basic model reveals essential trends with the bond coat yield strain, the imperfection size, the elastic properties of the TGO and the bond coat, the thermal expansion misfit, the TGO growth strain and the TGO thickness. The applicability of the model is justified by the correspondence in trends with simulations performed for representative non-spherical, imperfections and their association with experimental findings. A further justification is provided by the similarities with the elastic/plastic indentation of a half space.

The importance of the TGO yield strength emerges from the analysis, embodying a coupling with TGO growth and bond coat yielding. It is manifest in the contrast between multiple thermal cycles and a single cycle (isothermal) conditions for the same TGO growth strain. Namely, while there are no differences between the two histories when the TGO behaves elastically, thermal cycling causes substantially larger 


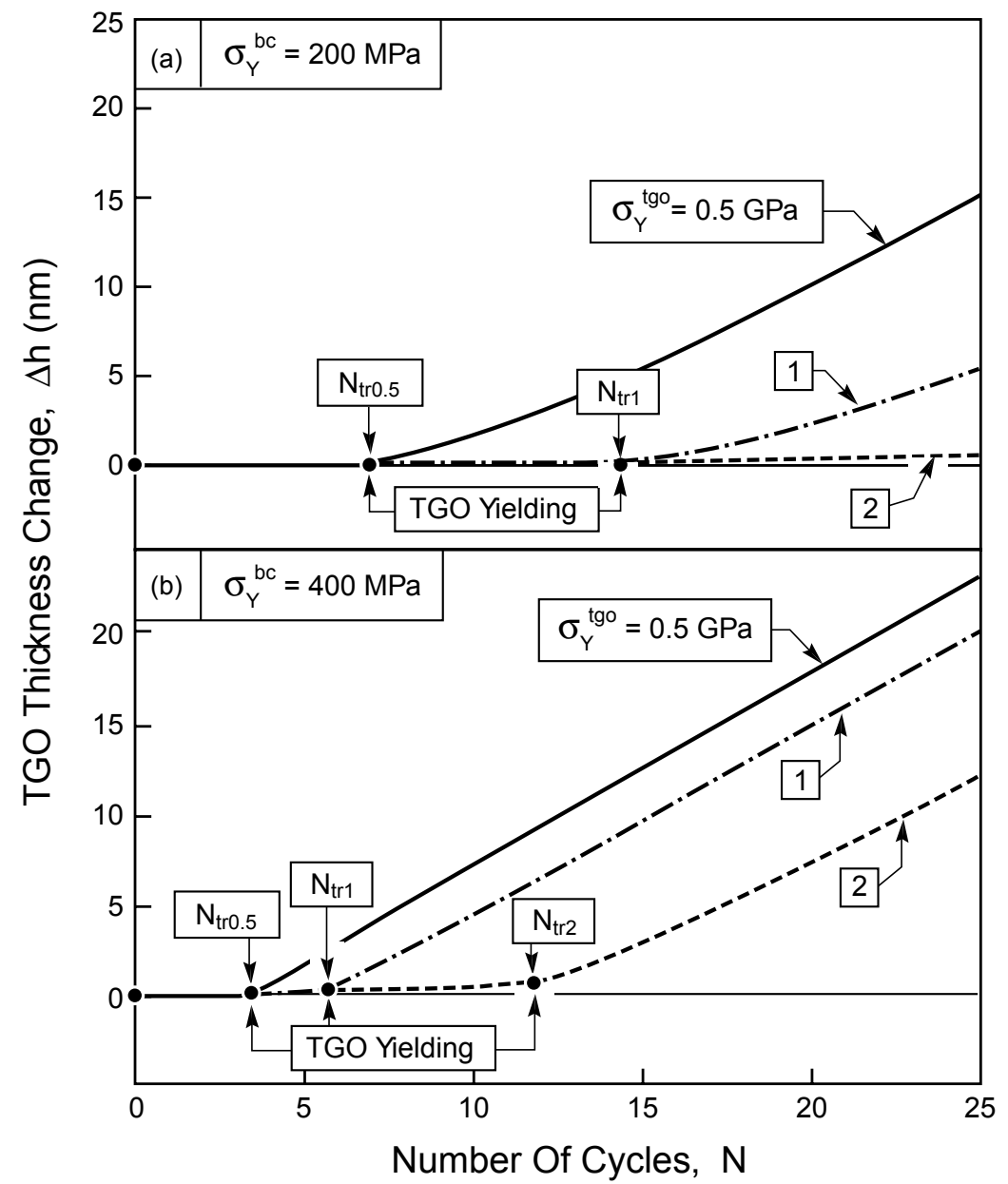

Fig. 16. The cycle-by-cycle thickening of the TGO for various TGO yield strengths: (a) $\sigma_{\mathrm{Y}}^{\mathrm{bc}}=200 \mathrm{MPa}$ (b) $\sigma_{\mathrm{Y}}^{\mathrm{bc}}=400 \mathrm{MPa}$. When TGO yielding occurs, the TGO thickness increases, even though the imposed thickening strain is zero.

radial enlargement when the TGO is allowed to yield at the peak temperature. This effect is fundamentally dictated by the absence of stress changes and of displacements once the TGO yields. That is, without cycling, after the TGO reaches yield, further TGO growth has no effect on the stresses. With temperature cycling, if the bond coat yields on cooling and a plastic zone develops, the stress in the TGO on reheating to the peak temperature is reduced (become less compressive) and additional TGO growth occurs prior to TGO yielding. Accordingly, when TGO growth is spread over multiple thermal cycles, a greater fraction takes place when the TGO is elastic, and the expansion of the TGO shell is greater. 
The model reveals that the instability is not dependent on reverse yielding of the bond coat, contravening an inference from earlier numerical simulations (He et al., 2000; Karlsson and Evans, 2001). However, reverse yielding is associated with low yield strength, and consequently does coincide with a rapid increase in the rate at which the instability enlarges.

The preceding results and implications can be used to interpret simulations of the growth of instabilities in actual thermal barrier systems, with the proviso that some circumferential displacements occur at actual imperfections, which are not allowed in the sphere model.

(i) Basic scaling tendencies with material properties can be ascertained directly from Eqs. (4) and (5).

(ii) The difference found between cyclic and isothermal responses can now be explicitly related to the in-plane growth strain in the TGO and its yield behavior.

(iii) The role of bond coat yielding is now more clearly connected to its effect on the stresses in the TGO upon cycling. There is no explicit requirement that it experience reverse yielding upon reheating.

\section{Appendix A. Development of radial stresses and strains in the bond coat}

An elaboration of the results in Fig. 12 is presented to facilitate understanding. First, consider the simulation for the elastic TGO (Fig. 12A). Starting from stress-free conditions at elevated temperature, the bond coat yields upon cooling and exhibits reverse yielding upon reheating (Start-A-B). Upon returning to the elevated temperature (B), growth strain is applied. When this proceeds isothermally, appreciable plastic strain accumulates (B-C) and the stress follows a plastic pathway governed by the bond coat. This pathway is continued during cooling (C-D) leading to additional plastic strain accumulations. The final reheating (D-End) initially responds elastically, and is accompanied by a limited amount of reverse yielding.

For multiple thermal cycles, the scenario changes after the initial cooling and heating sequence. Each subsequent cycle duplicates the preceding one, except for a shift to higher stress. On the figure, the response is exemplified by the 11 th cycle. The growth strain is applied between $\mathbf{1}$ and $\mathbf{2}$. It is sufficiently small that the bond coat remains elastic. Nevertheless, the strains introduced influence the accumulation of plastic strain associated with yielding upon cooling (2-3) and reverse yielding on reheating (3-4), resulting in the stress/strain shift from the start of the preceding cycle. Recall that there is no difference in the final state between single and multiple cycles with elastic TGO, since the same net transformation strain is applied (Section 3.4).

When the TGO yields (Fig. 12B), the responses change after the initial coolingheating sequence (Start-A-B). Now, when the growth strain is applied isothermally (B-C), the TGO yields before the bond coat. Once this happens, the additional growth strain is redistributed as TGO thickens (Fig. 16) and there are no further changes in the stress or strain in the bond coat, within the resolution of the figure (there is a small unresolved change governed by the thickening). This effect rationalizes the substantially lower stress and strain at $\mathbf{C}$, relative to the elastic TGO. 
For the cyclic scenario, the 11th cycle is again highlighted. Now, the preceding cooling/reheating phase of the cycle causes the TGO to be below yield when the next TGO growth step begins (1-2). Accordingly, until TGO-yield is reached, the addition of the TGO is able to increase the stress and the strain in the bond coat. This accounts for the extra strain per cycle relative to the isothermal scenario, leading to the appreciably larger final strain. Note that with further cycling, eventually, steps 1-2 will no longer allow the all stress in the TGO to diminish below yield. Thereafter, the only changes will be those associated with TGO thickening.

\section{References}

Ambrico, J.M., Begley, M.R., Jordan, E.H., 2001. Stress and shape evolution of irregularities in oxide films on elastic-plastic substrates due to thermal cycling and film growth. Acta Mater. 49, 1577-1588.

Begley, M.R., Evans, A.G., Hutchinson, J.W., 1999. Spherical impression of thin elastic films on elasticplastic substrates. Int. J. Solids Struct. 36 (18), 2773.

Begley, M.R., Mumm, D.R., Evans, A.G., Hutchinson, J.W., 2000. Analysis of a wedge impression test for measuring the interface toughness between films/coatings and ductile substrates. Acta Mater. 48, 3211-3220.

Bree, J., 1968. Incremental growth due to creep and plastic yielding of thin tubes subjected to internal pressure and cyclic thermal stresses. J. Strain Anal. 3, 122-137.

DeMasi-Marcin, J.T., Gupta, D.K., 1994. Surf. Coat. Technol. 68/69, 1.

Evans, A.G., Mumm, D.R., Hutchinson, J.W., Meier, G.H., Pettit, F.S., 2001. Mechanisms controlling the durability of thermal barrier coatings. Progr. Mater. Sci. 46, 505-553.

Gell, M., Vaidyanathan, K., Barber, B., Cheng, J., Jordan, E., 1999. Mechanism of spallation in platinum aluminide/electron beam physical vapor-deposited thermal barrier coatings. Metall. Mater. Trans. 30 A, 427-435.

He, M.Y., Evans, A.G., Hutchinson, J.W., 2000. The ratcheting of compressed thermally grown thin films on ductile substrates. Acta Mater. 48, 2593-2601.

He, M.Y., Hutchinson, J.W., Evans, A.G., 2002. Large deformation simulations of cyclic displacement instabilities in thermal barrier systems. Acta Mater. 50, 1063-1073.

Johnson, C.A., Ruud, J.A., Bruce, R., Wortman, D., 1998. Relationships between residual stress, microstructure and mechanical properties of electron beam physical vapor deposition thermal barrier. Surf. Coat. Technol. 109, 80-85.

Karlsson, A.M., Evans, A.G., 2001. A numerical model for the cyclic instability of thermally grown oxides in thermal barrier systems. Acta Mater. 49, 1793-1804.

Karlsson, A.M., Levi, C.G., Evans, A.G., 2002a. A model study of displacement instabilities during cyclic oxidation. Acta Mater. 50, 1263-1273.

Karlsson, A.M., Xu, T., Evans, A.G., 2002b. The displacement instability transition in thermal barrier systems. Acta Mater. 50, 1211-1218.

Miller, R.A., 1984. J. Am. Ceram. Soc. 67, 517.

Mumm, D.R., Evans, A.G., 2000. On the role of imperfections in the failure of a thermal barrier coating made by electron beam deposition. Acta Mater. 48, 1815-1827.

Mumm, D.R., Evans, A.G., Spitsberg, I., 2001. Characterization of a cyclic displacement instability for a thermally grown oxide in a thermal barrier system. Acta Mater. 49, 2329-2340.

Olsson, M., Giannakopoulos, A.E., Suresh, S., 1995. Elastoplastic analysis of thermal cycling-ceramic particles in a metallic matrix. J. Mech. Phys. Solids 43, 1639-1671.

Rebello, N., Levi, C.G., 2002, in preparation.

Ruud, J.A., Bartz, A., Borom, M.P., Johnson, C.A., 2001. Strength degradation and failure mechanisms of electron-beam physical-vapor-deposited thermal barrier coatings. J. Am. Ceram. Soc. 84, 1545-1552.

Spitsberg, I., Mumm, D.R., Evans, A.G., 2002. On the failure mechanisms of thermal barrier coatings with diffusion aluminide bond coatings. Submitted. 
Stiger, M.J., Yanar, N.M., Topping, M.G., Pettit, F.S., Meier, G.H., 1999. Z. Metall. 90, 1069-1078.

Strangman, T.E., 1985. Thin Solid Films 127, 93-105.

Tolpygo, V., Clarke, D.R., 2000. Surface rumpling of a (Ni, Pt)Al bond coat induced by cyclic oxidation. Acta Mater. 48, 3283-3293.

Wright, P.K., Evans, A.G., 1999. Mechanisms governing the performance of thermal barrier coatings. Curr. Opin. Solid State Mater. Sci. 4, 255-265. 